\title{
By invitation only?
}

\author{
The Nature Reviews Chemistry team welcome your proposals for review articles. We provide some \\ details here on what we like to see in a proposal and how we decide whether to proceed.
}

Writing a review is no small task. And done well, the potential for impact is at least as large as that for a research article
Betteridge's law of headlines states that any headline that ends in a question mark can be answered by the word no. The one here is no exception. While engaging with the chemistry community at conferences this past year, we have frequently been asked if unsolicited contributions to our journal are welcome. Although a large portion of our content is authored by those invited to write by our editorial team, the answer is a resounding yes.

We at Nature Reviews Chemistry strive to keep our readers abreast of the major developments in chemistry by inviting articles that cover all the subfields of chemistry. But doing so with a small team is a challenge, such that we are always happy to receive original and well-thought-out proposals. Indeed, we have already published some excellent reviews as a result of topics pitched to us directly by prospective authors.

With only limited space in our journal and a desire to cover all fields of chemistry, we must, like all the journals in the Nature Reviews family, be very selective. Careful planning of a review is required - regardless of whether it was an editor invitation or an author proposal that got the ball rolling. We must judge if a review is timely, if it has originality in its scope and insight, and if we expect that the author line-up can write authoritatively on the topic.

Given these criteria, we strongly advise prospective authors not to embark on writing a full draft of a review before contacting the editorial office. A short email enquiry about the suitability of a topic is a good place to start, and, should we feel the article has promise, we will likely request a synopsis. From this stage forward, consideration of invited reviews and unsolicited reviews follow the same path.

A synopsis is a summary of what authors aim to cover in an article, as well as why the topic is important and timely. Synopses vary in their exact content, but there are some characteristics that are common to the best ones:

A working title for the review - although a title may evolve during the writing process, it is good to have something in mind from the start. Although quirky or punny titles may seem appealing, the rise of the internet means that most articles are found using search engines rather than by scanning journal tables of contents. Quirky titles do not help in this regard and are usually funnier to the authors than they are to anyone else.
Author list and affiliations - in reviews, unlike primary research articles, significant value is attached to the authority of contributors and their research in the area of the proposed article. All listed authors must have agreed to participate in preparation of the review, and changes to the author list after submission require written agreement of all other co-authors.

Scope - it is important to define the scope of the review early in the writing process. Although simply starting to write may seem attractive, it often results in poorly focused reviews that can quickly become a rambling list of facts rather than a critical analysis of the literature.

Major subheadings of the review - just as important as defining the overall scope is thinking about how to break the topic down into cohesive sections. For each subheading, a few sentences or a short bulleted list of the major points that will be covered is helpful. Although plans may change during the writing process, estimating a word count for each section helps to ensure the final product is succinct and well balanced.

Conclusions of the article - in the past, we have noted that a good review is just as much about identifying problems and future directions for a field as it is about summarizing past achievements. Review articles, just like primary research articles, should have insightful conclusions that are supported by the preceding sections.

Proposed display items - it is not necessary to prepare any graphics at this stage, but it helps to consider the concepts that require illustration, where they will appear and whether a figure, table or box might work best. The brevity of our reviews means that you have to choose images that convey broad concepts or highlight the most notable examples.

Key references - an exhaustive list of works to be cited is not necessary, but a few references for each section of the text help us to judge balance, impact, coverage and, importantly, timeliness.

Writing a review is no small task. And done well, the potential for impact is at least as large as that for a research article. The feedback we have been getting is loud and clear - interesting, clear and insightful reviews are well received by our readership, who you should always keep in mind when writing. Maybe they will read your article and be engrossed in a new topic. Maybe they will see an old concept in a new light. And maybe they will take what they learn and apply it to some truly great new science. 